\title{
Sensory Prediction for Autonomous Robots
}

\author{
Ryo Saegusa, Francesco Nori, Giulio Sandini \\ Robotics, Brain and Cognitive Sciences Department \\ The Italian Institute of Technology, Italy \\ Email: ryos@ieee.org, \\ \{francesco.nori, giulio.sandini\}@iit.it
}

\author{
Giorgio Metta \\ LIRA-Lab, \\ University of Genoa, Italy \\ Email:pasa@liralab.it
}

\author{
Sophie Sakka \\ Laboratory of Solids Mechanics \\ University of Poitiers, France \\ Email: sophie.sakka@lms.univ-poitiers.fr
}

\begin{abstract}
For a complex autonomous robotic system such as a humanoid robot, the learning-based sensory prediction is considered effective to develop a perceptual environment model by itself. We developed a learning system for an autonomous robot to predict the next sensory information from the current sensory information and the expected action. The system we consider contains a learning procedure and a behavior generation procedure. The learning procedure uses a multi layer perceptron minimizing the error between a given sensory input and its predicted value. The behavior generation procedure is based on a uniform probablistic density function to sample the learning data randomly, which is the effective strategy when the system does not have any assumption or knowledge of the environment. We also investigated sensory blind prediction which should allow action plannning as well as offer a reliable forecast for a safe evolution of the robot in the environment. The simulation and experimental results show that the system learns interaction between the robot and the environment in high fidelity.
\end{abstract}

Index Terms-Sensing, Prediction, Autonomous robot, Learning, Environment perception

\section{INTRODUCTION}

Recently, the complexity of the autonomous robots as well as the tasks for the robots have increased gradually. Consequently, it becomes a hard work to develop autonomous robots to recognize the environment appropriately. The difficulty to implement artificial creatures is often discussed in the context of the frame problem [1], which points out that the developed creatures do not well deal with problems in the real world because of the infinite complexity of the problem setting.

In conventional studies of environment recognition for autonomous robots, the environment model and the reference patterns for recognition are well designed depending on the assumed tasks. However, when we assume general tasks in a complex environment, it becomes difficult to design a suitable environment model and reference patterns to perceive the real environment.

Learning is one of the effective strategies for autonomous robots to construct an environment model. Learning approaches are often applied to perceive the environment and to generate behaviors with vision or tactile sensing information [2]-[7].

Regarding learning with visual information, Bentivegna et al. exploited learning to determine an optimal way of assembling pre-defined primitives using visual feedback [2]. Nishide et al. realized the prediction of object dynamics from visual images [3]. Natale et al. exploited learning to improve accuracy in reaching objects [4]. Sun et al. proposed the realtime learning system for reaching problem and discussed the learning optimization to improve blind reaching [5].

In the field of learning with tactile information, Rucci et al. developed the learning approach of tactile-motor coodination and applied it to the active estimation of surface curvature [6]. Jamone et. al exploit learning for object classification with tactile sensing information measured at grasping the objects [7].

These conventional studies are effective in the case of environment modeling and behavior generation, however the learning sytems are specialized for some specific tasks and types of sensing.

This paper proposes a general sensory prediction system for autonomous robots based on learning. For autonomous robots, sensory prediction is considered as a primitive perception of the external world including its own existence, since it allows the robot to expect the effect of its own action in the environment and correct its expectation with the sensory information given later. We do not design any special environment model or reference patterns for the robots. Moreover, the sensory prediction is also useful to generate behaviors, since sensory prediction helps the robot deciding the next action.

In this paper, section II describes the proposed framework of sensory prediction. Section III describes the simulation with a physical environment model. Section IV reports the experimental results with observed data from a humanoid robot. Section V discusses the properties of the system and some ideas of improvements, and finnaly section VI concludes with possible extensions and future tasks.

\section{METHOD}

\section{A. Sensory prediction}

An autonomous robot must acquire an environmental model to act in the external world. Fig.1 illustrates the acquisition of an environment model for a robot. In Fig.1(a), a robot interacts with a real environment, while in Fig.1(b) the robot interacts with the acquired environment.

The idea addressed in this paper is to deal with the environment modeling as a sensory prediction problem. If the robot can predict a response of the environment for its action, this ability can be regarded as a perceptual environment modeling.

The sensory prediction is also effective to detect changes in the robot and the environment, since the sudden change 


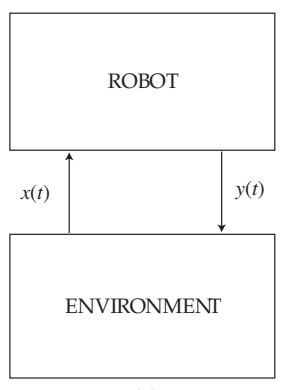

(a)

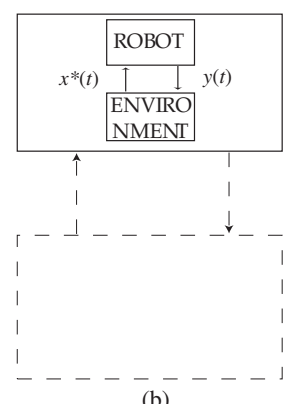

(b)
Fig. 1. Interaction between a robot and an environment. (a) Interaction with the real environment. (b) Interaction with the acquired environment model.

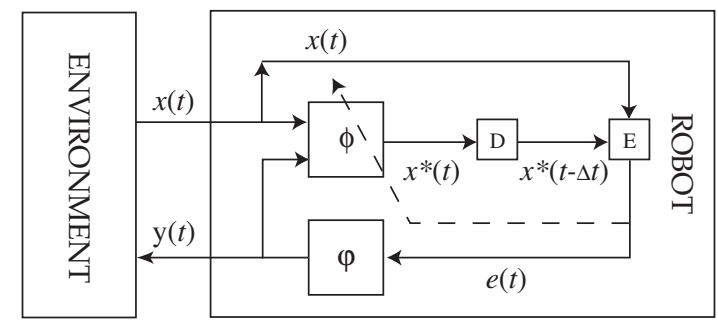

Fig. 2. Sensory prediction.

of error indicates that the environment or its body has been modified. Therefore, the sensory prediction is applicable to dynamical environment perception and self-diagnosis.

The sensory prediction is defined as shown in Fig.2 using the following equations.

$$
\begin{aligned}
\vec{x}^{*}(t) & =\phi(\vec{x}(t), \vec{y}(t)), \\
\vec{y}(t) & =\psi(e(t)),
\end{aligned}
$$

where $\vec{x}(t)$ and $\vec{y}(t)$ are vectors grouping sensor and motor information at time $t$, respectively. $\vec{x}^{*}$ represents prediction of the next sensory information at time $t$ and $e(t)$ is the prediction error at time $t . \phi(\cdot)$ and $\psi(\cdot)$ are arbitrary mapping functions for sensory prediction and motor actuation, respectively. The operator $D$ in Fig. 2 indicates time delay, and it aims at keeping the value of prediction at time $t$ until the next discrete time $(t+\Delta t)$. The operator $E$ in Fig.2 calculates the prediction error.

Here, the sensory information is assumed to include both the internal and external sensing. In robotics, kinesthesis sensing and somatosensing are regarded as internal sensing, while the visual, auditorial or tactile sensing are regarded as external sensing. In this framework, we do not differenciate these two categories of sensing from the viewpoint of generality. We will focus on the cognitive part of the problem, which consists in establishing a model between sensory information and motor control.

The prediction error at time $t$ is defined as the squared Euclidean distance using the following equation,

$$
e(t)=\left\|\vec{x}^{*}(t-\Delta t)-\vec{x}(t)\right\|^{2} .
$$

The error is based on the difference between the next sensory

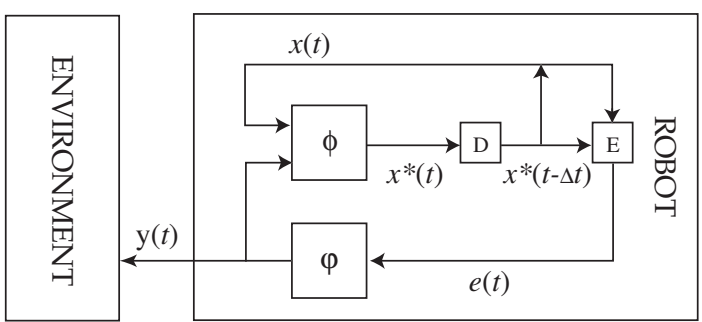

Fig. 3. Sensory blind prediction.

input predicted at time $(t-\Delta t)$ and the real sensory input measured at time $t$.

The prediction function $\phi(\cdot)$ is optimized based on this error through learning. The function is evaluated inside the system. Therefore, this learning is regarded as an unsupervised learning from the outside.

The behavior of the robot is given by the actuation function $\psi(\cdot)$. In general, the behavior should be designed for the tasks given to the robots. In this framework, we define the robot's task as to interact with the environment in order to obtain learning data for the sensory prediction.

Consequently, the processing procedure is summarized as follows,

1) Acquire the sensory input $\vec{x}(t)$.

2) Evaluate the last prediction error $e(t)$.

3) Modify the prediction function $\phi(\cdot)$.

4) Generate the next action $\vec{y}(t)$.

5) Predict the next sensing $\vec{x}(t)$.

6) Perform the motor output $\vec{y}(t)$.

\section{B. Sensory blind prediction}

Sensory prediction system works as a short term prediction, since the system predicts the next sensory information based on the current sensory information and the expecting action. The autonomous robot, however, needs to see the environment in perspective, when it plans its own action. Sensory prediction system allows this long term prediction, if the system successively feedbacks its own sensory prediction $\vec{x}^{*}(t)$ as the next sensory input, instead of the real sensory input $\vec{x}(t)$ from the environment. We call this procedure sensory blind prediction. It is illustrated in Fig. 3 using the following definition,

$$
\begin{aligned}
\vec{x}(t+\Delta t) & =\vec{x}^{*}(t), \\
& =\phi(\vec{x}(t), \vec{y}(t)) .
\end{aligned}
$$

In the sensory blind prediction, the system can generate an expected sequence of the sensory input in the near future. However, the prediction error between the predicted variables and the real variables accumulates through many iterations of feedbacks. Consequently, the values of the predicted sensory variables gradually lose reliability as sensory blind prediction is iterated. The other reason of losing reliability is related to the absence of learning during sensory blind prediction, since the target signals are not given from the environment. However, if the sensory prediction system is well trained, 


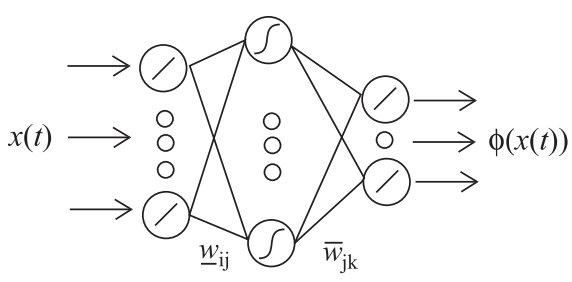

Fig. 4. Multi Layer Perceptron (MLP).

sensory blind prediction is reliable during some iterations and effective for the action planning.

\section{Implementation by neural networks}

The mapping function $\phi(\cdot)$ for the sensory prediction was implemented with Multi Layer Perceptron (MLP) as shown in Fig.4. MLP is an universal function approximator, whose parameters can be optimized by learning. We adopted the MLP with three layers and the learning strategy based on the gradient method [9]. The MLP with three layers can approximate any function in any accuracy, depending on the number of hidden units in the second layer.

Here, the prediction function $\phi(\cdot)$ is defined as follows,

$$
\phi_{k}(\vec{x})=\sum_{j=1}^{\bar{n}} \bar{w}_{j k} \cdot f_{a}\left(\sum_{i=1}^{\underline{n}} \underline{w}_{i j} x_{j}+\underline{w}_{0 j}\right)+\bar{w}_{0 k}
$$

where $\phi_{k}(\cdot)$ represents the $k$-th component of the funtion $\phi(\cdot)$. $\bar{w}_{j k}$ are the weight coefficients connecting the first to second layer, and $\underline{w}_{i j}$ connecting the second to third layer. $\bar{w}_{0 k}$ and $\underline{w}_{0 j}$ are bias coefficients. $\underline{n}$ and $\bar{n}$ are the numbers of the units in the first and second layer, respectively. As shown in Fig.4, the activation function $f_{a}$ of the units in the second layer is a differentiable non-linear function, while the activation functions $f_{a}$ of the units in the first and the third layers are identical functions. We adopted the hyperbolic tangent as $f_{a}$ in the second layer as follows.

$$
f_{a}(u)=\tanh \left(\frac{u}{T}\right),
$$

where $T$ is a constant value to control non-linearity and $u$ is a weighted sum of the inputs into the units.

The parameters of the function $\underline{w}_{i j}$ and $\bar{w}_{j k}$ are modified for each input $\vec{x}(t)$ to minimize the error $e(t)$ using gradient descent:

$$
\Delta \underline{w}_{i j}=-\eta \frac{\partial e}{\partial \underline{w}_{i j}}, \quad \Delta \bar{w}_{j k}=-\eta \frac{\partial e}{\partial \bar{w}_{j k}},
$$

where $\eta$ is a constant learning rate.

The actuation function $\psi(\cdot)$ is implemented as a stochastic function. The stochastic strategy is effective for sampling of learning data, when the system does not have any assumption or knowledge of the environment. We adopted the uniform probablistic density function $p d f(u)$ for the random variable of $u$.

$$
p d f(u)= \begin{cases}\frac{1}{2 r} & \text { for }|u| \leq r, \\ 0, & \text { for }|u|>r,\end{cases}
$$

where the constant $r$ represents the range of the variable $u$.

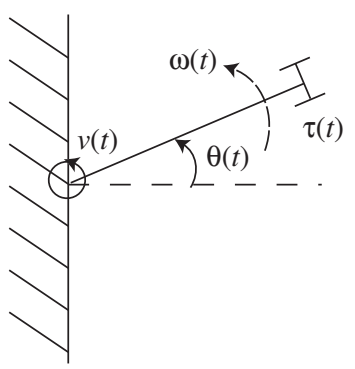

Fig. 5. Environment for simulation.

\section{Simulation}

We performed a simulation to evaluate the two proposed sensory prediction systems using the physical environment model.

\section{A. Simulation setting}

Let us consider a one-joint arm set on a flat wall as the environment as illustrated in Fig.5. The arm and wall are constrained on a horizontal plane. The end effector of the arm is equiped with a tactile sensor. The problem simulates elastic collision between the arm and the wall, therefore the range of the arm's joint rotation is limited in $[-\pi / 2,+\pi / 2]$ degree. The variables of sensor and motor are defined as follows,

$$
\begin{aligned}
& \vec{x}(t)=(\theta(t), \omega(t), \tau(t)), \\
& \vec{y}(t)=(v(t)),
\end{aligned}
$$

where $\theta(t), \omega(t), \tau(t)$ refer to the joint angle, joint angular velocity and tactile sensing, respectively. $v(t)$ is the voltage for the motor actuation.

The dynamics of the arm between the time $t$ and $(t+\Delta t)$ are defined with two sets of equations regarding collision. The dynamics without collision is defined:

$$
\begin{gathered}
\theta(t+\Delta t)=\theta(t)+\omega \Delta t+\xi, \\
\omega(t+\Delta t)=\omega(t)+f_{r}(v(t)) \Delta t+\xi, \\
f_{r}(v)= \begin{cases}c\left(v-v_{\text {inf }}\right), & \text { for } v \leq v_{\text {inf }}, \\
0 & \text { for } u_{\text {inf }}<v<v_{\text {sup }}, \\
c\left(v-v_{\text {sup }}\right), & \text { for } v_{\text {sup }} \leq v,\end{cases}
\end{gathered}
$$

where $f_{r}(\cdot)$ is a non-linear function which models the static friction of the joint motor. The function includes a refractory domain. $\xi$ simulates small triangular noise to represent the external disturbance.

The dynamics at collision is defined as,

$$
\begin{aligned}
& \theta(t+\Delta t)=\theta(t), \\
& \omega(t+\Delta t)=-e_{l} \omega(t),
\end{aligned}
$$

where the $e_{l}$ represents the elastic coefficient. The state of the arm is regarded as collision, if the absolute value of the next joint angle $|\theta(t+\Delta t)|$ calculated by the Eq.(13) is over $\pi / 2$. In this case, the next values of joint angle and joint angular velocity are replaced by the values calculated by the Eq.(18) and (19), respectively. 


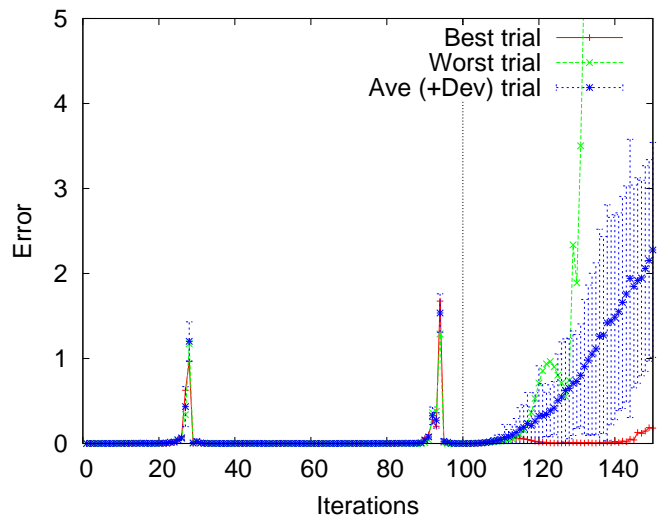

Fig. 6. Error of the sensory prediction and sensory blind prediction averaged on 100 trials.

The tactile sensing is represented as follows,

$$
\tau(t)= \begin{cases}\tau_{\text {inf }}, & \text { at the collision, } \\ \tau_{\text {sup }}, & \text { otherwise }\end{cases}
$$

where $\tau_{\text {inf }}$ and $\tau_{\text {sup }}$ are constant. This on-off response is perceived as non-linear by the prediction system.

We performed sensory prediction of the above mentioned problem. In the simulation, $\Delta t$ and $e_{l}$ were set to $50 \mathrm{~ms}$ and 0.8 , respectively. $v_{\text {inf }}$ and $v_{\text {sup }}$ were -0.2 and +0.2 volt, while $\tau_{\text {inf }}$ and $\tau_{\text {sup }}$ were -1.0 and $+1.0 . c$ was set to $0.1 . r$ was set to 1.0. The input values of $\theta$ and $\omega$ for the prediction sytem were normalized between $[-1.0,+1.0]$ to stabilize the learning. The number of the units in the second layer of MLP was set to 30. $T$ and $\eta$ were set to 1.0 and 0.01 . All the weight coefficients of MLP were randomized between $[-1.0,+1.0]$. The learning was iterated 5,000,000 times. Each trial of the learning completed less than 20 seconds on a laptop computer mounted with dual $2.0 \mathrm{GHz}$ CPUs. The learning was performed and followed by the sensory blind prediction. The sensory blind prediction was iterated 50 times. In order to offer a reliable analyze of the algorithms behavior, we will discuss the results on an average of 100 simulations, also showing the best and worst solutions.

\section{B. Learning results}

The error in the 150 iterations of the sensory prediction is shown in Fig.6. The first 100 iterations correspond to the sensory prediction with learning. Before the iterations, the long learning was performed as mentioned above. The last 50 iterations correspond to the sensory blind prediction.

The trial is evaluated by the mean squared error (MSE) as follows.

$$
M S E=\frac{1}{k_{e}-k_{s}} \sum_{k_{s} \leq k<k_{e}} e(t+k \Delta t),
$$

where $k_{s}$ and $k_{e}$ correspond to the 0 -th and 150-th iteration in the Fig.6, respectively.

As shown in the figure, the sensory prediction was on average well performed. The prediction error remains under
$10^{-4}$ until the 100-th iteration except for two peaks caused by collision. At the 100-th iteration, the sensory blind prediction started. As we are observing average resutls, we can insure with this algorithm an acceptable prediction, during 10 iterations. The error after the period, however, gradually increased in the higer order. The error was amplified through many times of blind feedbacks which included the temporal error. The error in the best trial was still small until the 140th iteration, approximately, while the error in the worst trial exponentially increased. The trials showing greater error may be caused by the failure in finding a global minimum when optimizing the value of the weights, either for the learning or for the blind prediction. In such case, the values are trapped in a local minimum and this effects the performance of the algorithm. However, the error value during learning sticks to zero for all this simulations, which indicates that the value of the weights were correctly optimized.

Fig.7 compares the time variations of the predicted sensory variables, $\theta(t) \omega(t)$ and $\tau(t)$ and their real values. The predicted variables of the best trial are close to the real sensory variables, however the ones of the worst trial diverged after the blind prediction started. Through these figures, we can easily notice the non-linear dynamics in this problem. Around the 30-th and 90-th, the supposed arm and wall make the elastic collision. However, the system well predicted this non-linear dynamics and tactile reaction at these collisions as shown in Fig.7(c) of $\tau(t)$. The influence of the elastic collision also appears in the Fig.6 at the peaks of the error.

\section{EXPERIMENT}

We applied the proposed system to predict sensory information of a humanoid robot.

\section{A. Experiment setting}

The experiment was performed using the joint data sampled from the shoulder motor of the humanoid robot James [7]. The Fig. 8 shows the appearance of James. James consists of 22 degrees of freedom, actuated by 23 motors. The torques of the motors are transmitted to the joints by belts and stainlesssteel tendons. The design of the tendon driven shoulder was developed to allow wide-range movements. Actuation is achieved by the tendons and pulleys.

The variables of sensor and motor are defined as follows,

$$
\begin{aligned}
& \vec{x}(t)=(\theta(t), \omega(t)), \\
& \vec{y}(t)=(v(t)),
\end{aligned}
$$

where $\theta(t)$ and $\omega(t)$ indicate the angle and angular velocity of the shoulder joint. $v(t)$ is the voltage for the motor actuation. It is worthy of remark that tactile sensing is not included in this experiment because of the current hardware setting of the humanoid robot. $\theta(t)$ and $\omega(t)$ are both sensory information detected in the internal environment by the encoders, namely the robot's body. Here, we do not differentiate the source of sensory information as mentioned before. 

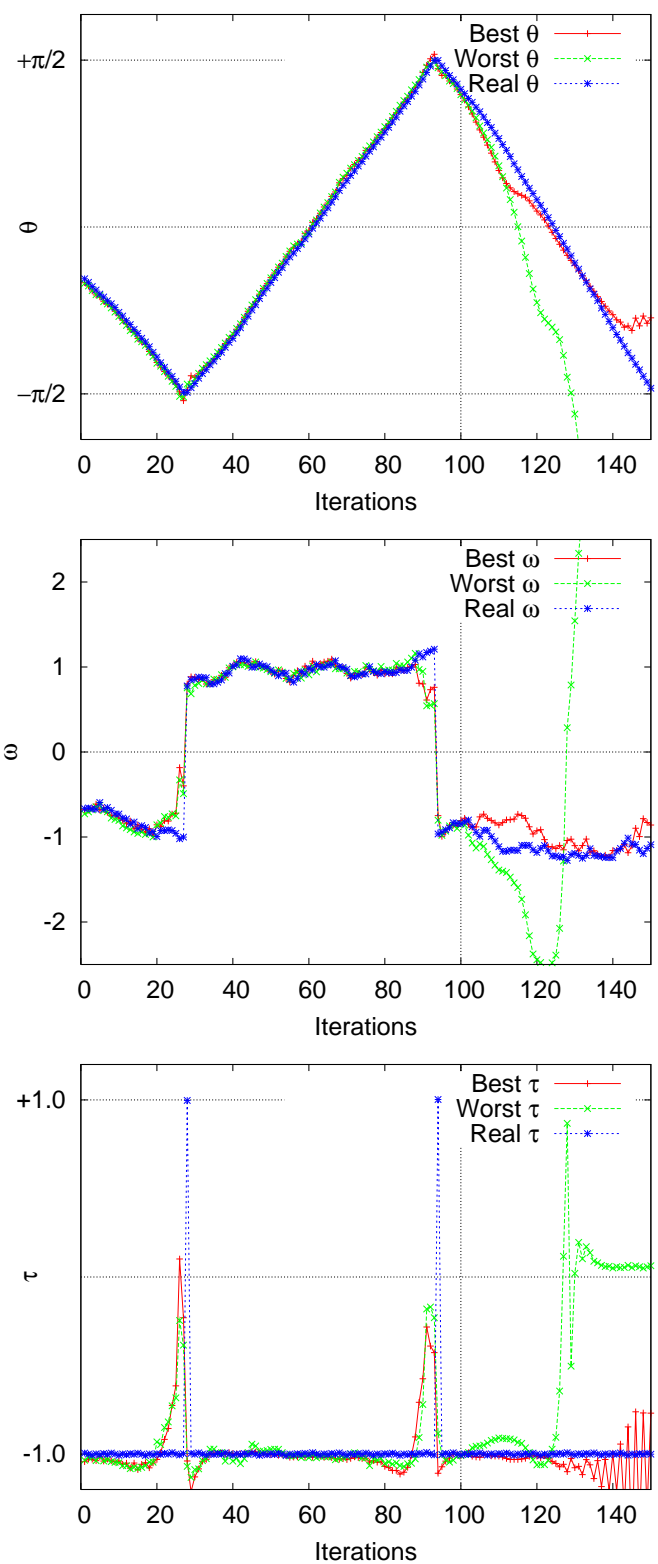

Fig. 7. Time variations comparison of predicted and real sensory variables. (a) Joint angle $\theta(t)$, (b) Joint angular velocity $\omega(t)$, (c) Tactile sensing $\tau(t)$.

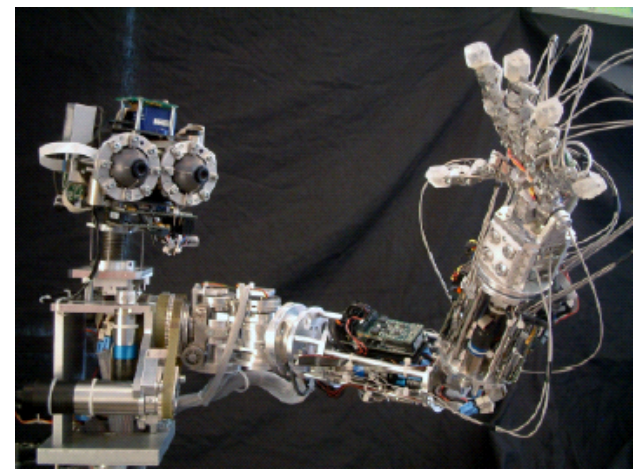

Fig. 8. Humanoid robot James [7].

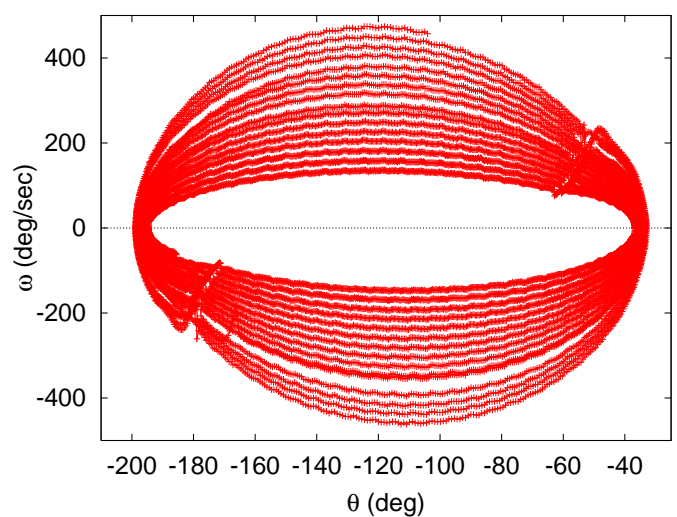

Fig. 9. The sampled trajectory on the phase plane.

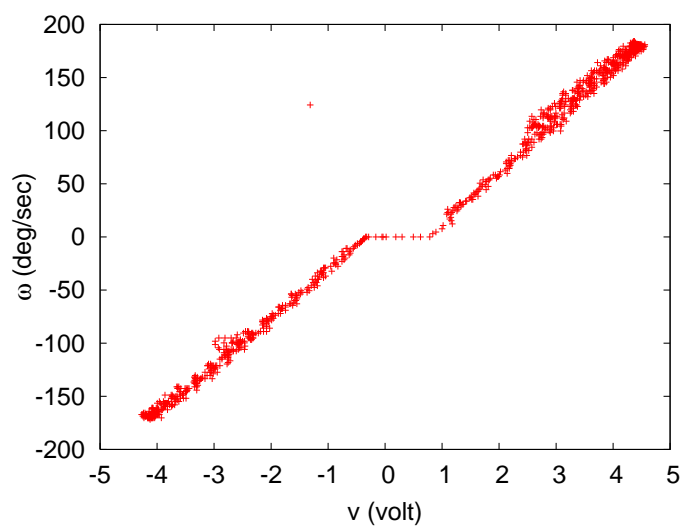

Fig. 10. Motor characteristics

Firstly, we observed the angle $\theta(t)$ with controling the value of $v(t)$. Then, $\omega(t)$ was calculated from the observed $\theta(t)$ by the following time derivation,

$$
\omega(t)=\frac{1}{\Delta t}\{\theta(t)-\theta(t-\Delta t)\} .
$$

The temporal pattern of $v(t)$ is designed experimentally to make the trajectory of $(\theta(t), \omega(t))$ cover the larger area on the phase plane. The sampled trajectory is shown in Fig.9, leading to the motor characteristics shown in Fig.10, which suggests that the static friction is loaded at the motor driving. The motor response for the control is piecewise linear.

\section{B. Experimental results}

We performed sensory prediction of the joint data. Prior to the experiments, the joint data were sampled. The sampling interval of the data $\Delta t$ was set to $2 \mathrm{~ms}$. The number of the sampling points is about 65,000 . Therefore, the total sampling time corresponds to 130 seconds approximately. According to the hardware design, the range of the joint angle $\theta(t)$ was limited in $[-200,-20]$ degree, and the range of the voltage $v(t)$ was limited in $[-12,+12]$ volt.

After the data sampling, we extracted a sequence of 15,000 points to remove the apparent noise. Each point of the sequence corresponds to $\{\vec{x}(t), \vec{y}(t)\}$. The randomly selected 


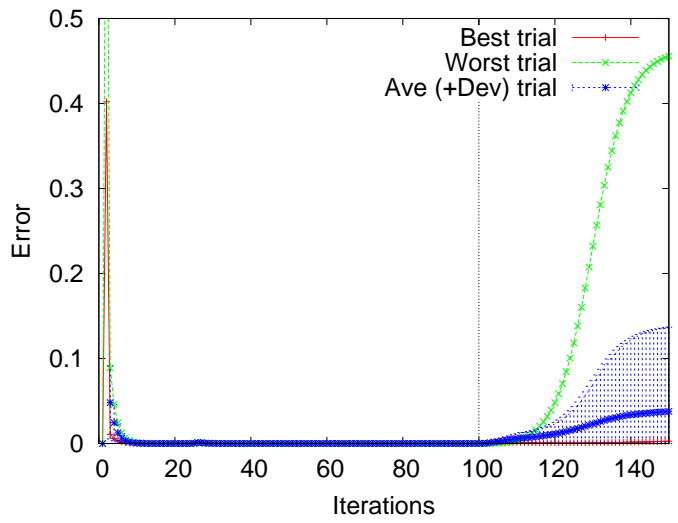

Fig. 11. The error of the sensory prediction.

1,000-length sequences were used as the interaction data between the robot and the environment. Therefore, the voltage output $\vec{y}(t)$ was fixed for the encoder input $\vec{x}(t)$, however, the short sequence of these coupled data $\{\vec{x}(t), \vec{y}(t)\}$ were randomly selected and given to the sensory prediction system.

In the experiment, the learning was iterated 1,000,000 times. The other conditions were same as the ones in the simulation before. Each trial of the learning completed less than 25 seconds on a laptop computer mounted with dual $2.0 \mathrm{GHz}$ CPUs.

The value with time error in the 150 iterations of the sensory prediction is shown in Fig.11. The first 100 iterations and the last 50 iterations correspond to the usual and blind prediction, respectively. As shown in the figure, the sensory prediction was on average well performed. The experimental result is very similar to the simulation with physical environment model. During the usual sensory prediction, the error was approximately zero, while during the sensory blind prediction, the error was amplified. The large error at the begining of the iteration was caused by the switching of the given sequence data. It is not important in this discussion.

The Fig.12 shows the time variations of the predicted sensory variables. The predicted variables follow the real sensory variables well. As mentioned above, the motor has the non-linear characteristics in driving. However, the sensory prediction system functioned as expected.

\section{Discussion}

The experimental results suggests that the learning-based sensory prediction system is effective for autonomous robots to develop a perceptual environment model. Here, we discuss some ideas of improvements to allow this sytem to work on a more complex platform such as a full-bodied humanoid robot.

\section{A. Sensory information coding}

Spatio-temporal coding of sensory information seems effective to improve the quality of prediction. The current system predicts physical sensory information such as position, velocity and contact. The physical sensory prediction makes sense, since dynamics of these variables can be physically
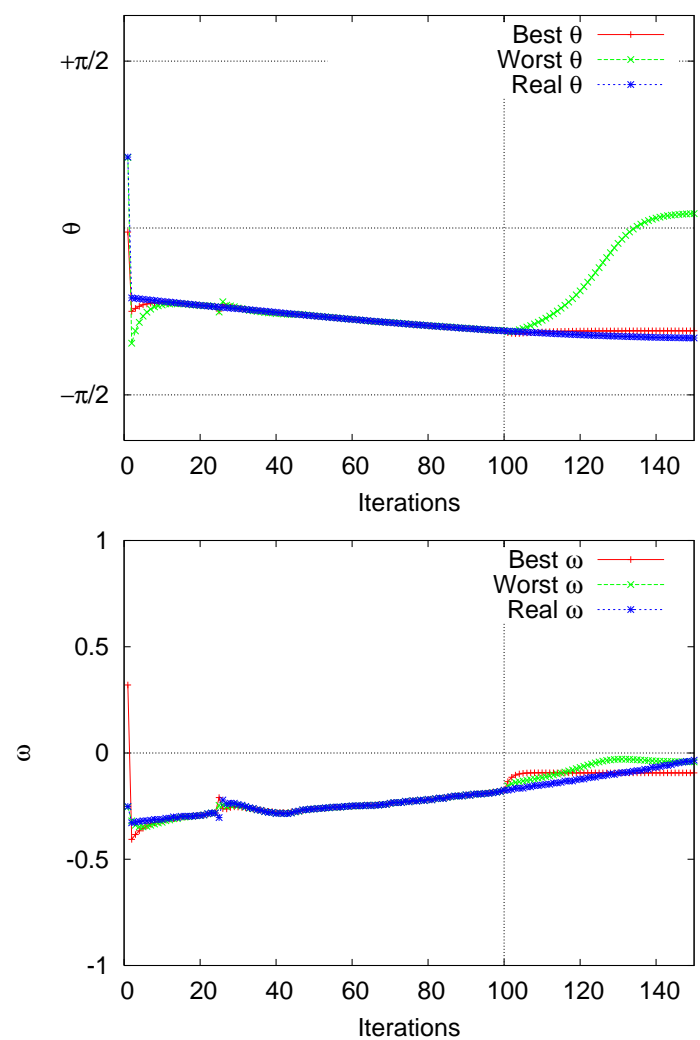

Fig. 12. Time variations of the predicted sensory variables. (a) Joint angle $\theta(t)$, (b) Joint angular velocity $\omega(t)$.

described as spatio-temporal differential equations. In order to generate actions of an autonomous robot, however, it is too enough to predict all of the physical variables. The prediction of summarized or coded sensory information seems more suitable. The perceptual sensory prediction is effective rather than physical sensory prediction. The coding benefits the robot to reduce computational costs toward real time learning. The coding is also effective for long term prediction. It would make higher reliability in sensory blind prediction.

\section{B. Behavior generation for active learning}

The advantage of the embodied learning system is that the learning system can exploit its mobility to collect learning data actively. In the conventional framework of learning, the learning system was passive for data collection. The proposed sensory prediction system, however, can generate its action to collect data for learning improvement. The sensory prediction system can recognize weak parts of learning by prediction error and generate actions to compensate these weak parts. As shown in the experimental results, the level of difficulties in learning depends on situations. In the simulation of physical environment model, dynamics of variables at collision was difficult to predict. The active learning strategy allows the system to learn this part specially by generating collision many times. 


\section{CONCLUSION}

We proposed a sensory prediction system for autonomous robots based on a learning procedure and an activation procedure. The sensory prediction system predicts the next sensory information from the current sensory information and the expected action. In this paper, we implemented classical prediction and blind prediction. The first one uses external real values as an input, and the second one uses its own knowledge. The simulated results show that the system learns the interaction between the robot and the environment in high fidelity, when using external source of information. This enables the system to predict sensory information correctly and blindly in a limited number of iterations.

The sensory blind prediction is an effective tool for interpolating sensory information, when learning data is not available during a short time. We pointed out that whatever the situation, blind prediction allows to predict safelly for the 10 time steps following an absence of learning data.

Even though the sensory information is temporally unavailable, the blind prediction suggests the next situation of the robot and the environment. The blind prediction is also effective to anticipate the results of action sequences, which enables the robot to compare several scenarios of the behavior and to select the best. These abilities contribute to the safety of both the robot and the environment to avoid an accident by predicting it.

Now that the effectiveness of our approach was shown, we have to focus on several tasks aimed at improving the results of this study: we mentioned that blind prediction would be an asset to interpolate sensory information. Going through the interpolation procedure using both usual and blind prediction shall be the first task to perform, as well as improving the performance of the blind prediction.

We are assuming to mount the proposed sensory prediction system on a humanoid robot. The expected tasks are tapping behavior to percept the environment spatially for real-time action.

\section{ACKNOWLEDGMENT}

The work presented in this paper has been patially supported by the ROBOTCUB project (IST-2004-004370), funded by the European Commission through the Unit E5 "Cognitive Systems". The authors would like to thank Prof. Shuji Hashimoto in the department of applied physics, Waseda university for his helpful suggestions throughout this paper.

\section{REFERENCES}

[1] J. McCarthy, P. J. Hayes, "Some philosophical problems from the standpoint of artificial intelligence," Machine Intelligence 4, pp.463-502, 1969.

[2] D. C. Bentivegna, C. G. Atkeson, A. Ude, G. Cheng, "Learning to act from observation and practice," International Journal of Humanoid Robotics, vol.1 No4, pp 585-611, 2004.

[3] S. Nishide, T. Ogata, J. Tani, K. Komatani, H. G. Okuno, "Predicting object dynamics from visual images through active sensing experiences," Proc. of 2007 IEEE International Conference on Robotics and Automation, Roma, Italy, 10-14 April 2007.

[4] L. Natale, F. Nori, G. Sandini, G. Metta, "Learning precise 3D reaching in a humanoid robot," ICDL 2007. London, UK. July 11-13, 2007.
[5] G. Sun, B. Scassellati, "A fast and efficient model for learning to reach," International Journal of Humanoid Robots, vol 2, no4, pp. 391-413, 2005.

[6] M. Rucci and P. Dario, "Autonomous learning of tactile-motor coordination in robotics," Proc. of 1994 IEEE International Conference on Robotics and Automation, 8-13 May 1994, pp. 3230-3236 vol.4, 1999.

[7] L. Jamone, G. Metta, F. Nori and G. Sandini, "James, a humanoid robot acting over an unstructured world," Proc. of the Humanoids 2006 conference, December 4-6th, 2006, Genoa, Italy, 2006.

[8] L. Natale, E. Torres-Jara, A sensitive approach to grasping. Sixth international Conference on Epigenetic Robotics, Paris, France, 20-22 September, 2006.

[9] D. Rumelhart, J. McClelland, "Learning internal representation by error propagation," Parallel Distributed Processing, pp. 318-362, MIT Press, 1984.

[10] M. Kawato, "Internal models for motor control and trajectory planning," Current Opinion in Neurobiology, 9, pp. 718-727, 1999.

[11] D. M. Wolpert, Z. Ghahramani, R. J. Flanagan, "Perspectives and problems in motor learning," Trends in Cognitive Sciences, 5(11), pp.487494, 2001.

[12] D. M. Wolpert, R. C. Miall, 'Forward models for physiological motor control," Neural Networks, 9(8), pp.1265-1279, 1996.

[13] R. D. Beer, "Dynamical approaches to cognitive science," Trends in cognitive sciences, 4(3), pp.91-99, 2000.

[14] M. Haruno, D. M. Wolpert, M. Kawato, "MOSAIC Model for sensorimotor learning and control," Neural Computation, 13, pp.2201-2220, 2001.

[15] M. I. Jordan, D. E. Rumelhart, 'Forward models: Supervised learning with a distal teacher," Cognitive Science, 16(3), pp.307-354, 1992. 